\title{
BMJ Open Human papillomavirus prevalence and genotype distribution among young women and men in Maputo city, Mozambique
}

Viegas Edna Omar, ${ }^{1,2,3}$ Augusto Orvalho, ${ }^{3}$ Ismael Nália, ${ }^{1}$ Kaliff Malin, ${ }^{4}$
Lillsunde-Larsson Gabriella, ${ }^{4}$ Ramqvist Torbjörn, ${ }^{5}$ Nilsson Charlotta, ${ }^{2,6,7}$
Falk Kerstin, ${ }^{6,7}$ Osman Nafissa,,${ }^{3,8}$ Jani Ilesh Vindorai, ${ }^{1}$ Andersson Sören ${ }^{4}$

To cite: Edna Omar V, Orvalho A, Nália I, et al. Human papillomavirus prevalence and genotype distribution among young women and men in Maputo city, Mozambique. BMJ Open 2017;7:e015653. doi:10.1136/ bmjopen-2016-015653

- Prepublication history for this paper is available online. To view these files please visit the journal online (http://dx.doi. org/10.1136/bmjopen-2016015653).

Received 21 December 2016 Revised 10 May 2017 Accepted 11 May 2017

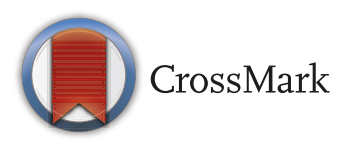

For numbered affiliations see end of article.

Correspondence to Dr Viegas Edna Omar; ednaviegas@gmail.com

\section{ABSTRACT}

Objectives Human papillomavirus (HPV) is a well-known cause of cervical cancer, the second most frequent cancer in female African populations. This study aimed at determining the prevalence of HPV infections and the genotype distribution in young adults aged 18-24, in Maputo city, Mozambique, and to assess the suitability of commercially available HPV vaccines.

Methods This cross-sectional study was conducted between 2009 and 2011 at a youth clinic in Maputo Central Hospital. Cervical and urethral samples were obtained from 236 women and 176 men, respectively. Demographic and behavioural data were collected using structured questionnaires. HPV genotyping was performed for 35 different high, probably or possibly high-risk and low-risk HPV types using the CLART Human Papillomavirus 2.

Results HPV prevalence was 168/412 (40.8\%; 95\% Cl 36.0 to 45.5$)$ and was significantly higher in women than in men $(63.6 \%$ vs $10.2 \%)$. HPV52 was the most frequent type found in women, followed by HPV $35,-16,-53$, $-58,-6$ and -51 . In men, HPV51 ranked the highest, followed by HPV6, $-11,-52,-59$ and -70 . HIV infection and sexual debut before 18 years of age were associated with multiple HPV infections (OR $3.03 ; 95 \% \mathrm{Cl} 1.49$ to 6.25 and $\mathrm{OR} 6.03 ; 95 \% \mathrm{Cl} 1.73$ to 21.02 , respectively). Women had a significantly higher HPV infection prevalence than men $(p<0.001)$. The 9 -valent HPV vaccine would cover $36.8 \%$ of the high-risk genotypes circulating in women in this study, compared with $26.3 \%$ and $15.8 \%$ coverage by the bivalent and quadrivalent vaccines, respectively. Conclusion This study confirmed the high burden of HPV infections in young women in Maputo city, Mozambique. The HPV prevalence was associated with high-risk sexual behaviour. Sex education and sexually transmitted infection prevention interventions should be intensified in Mozambique. Only a proportion of the high-risk HPV genotypes $(37 \%)$ were covered by currently available vaccines.

\section{INTRODUCTION}

Globally, cervical cancer (CC) is the fourth most common cancer in women and the second most common cancer in female
Strengths and limitations of this study

- This is the first study to describe the human papillomavirus (HPV) prevalence and genotype distribution in young adults (aged 18-24) in Mozambique.

- This study provides insights on the HPV types circulating in young populations in Maputo city, which is an important public health information particularly for discussions on HPV vaccine introduction in the country.

- The absence of an older cohort (aged $\geq 25$ years) for comparison of circulating HPV genotypes was a limitation of this study.

- The lower HPV prevalence in the male population may have been related to the site of sample collection, therefore additional studies in male population in Mozambique, using different anatomic collection sites should be considered.

\section{Key messages}

There is a high burden of human papillomavirus (HPV) infection in young women in Maputo city, Mozambique.

- High-risk sexual behaviour was demonstrated to be associated with high prevalence of HPV infection.

- There is a need to intensify sex education and prevention of sexually transmitted infections in young adults.

- Currently available HPV vaccines would only cover a proportion $(37 \%)$ of the prevalent high-risk genotypes.

African populations. ${ }^{1}$ More than $75 \%$ of cases occur in less developed areas. East Africa is the most affected region with an age-standardised incidence rate of 42.7 per 100000 women. Mozambique has the second highest incidence of CC after Malawi. ${ }^{2}$

Human papillomavirus (HPV) infection has been implicated in virtually all CC cases. 
To date, 201 different HPV types have been identified. More than 40 of these types can infect the anogenital tract and have been classified as low-risk (lrHPV6, -11 , $-40,-42,-43,-44,-54,-61,-62,-71,-72,-81,-83,-84$ and -89 ), probably or possibly high-risk (phr-HPV26, -30 , $-34,-53,-66,-67,-68,-69,-70,-73,-82,-85$ and -97$)$ and high-risk HPV types (hr-HPV16, $-18,-31,-33,-35$, $-39,-45,-51,-52,-56,-58$ and -59$)$ depending on their ability to lead to malignant progression. ${ }^{4-6}$

Worldwide, HPV16 has been responsible for more than half $(54.4 \%)$ of the CC cases followed by HPV18 (16.5\%), HPV58 (5.1\%), HPV33 (4.7\%), HPV45 (4.4\%), HPV31 $(3.6 \%)$, HVP52 (3.4\%) and HPV35 (1.9\%). ${ }^{7}$ However, only a small proportion of infected women develop the disease. Persistent infection with hr-HPV types and integration of HPV-DNA in the host cells are usually required for malignant transformation of the cervix. The time between infection and initial precancer lesions is around $7-10$ years. Cervical cancer is more frequently diagnosed in middle-aged women (40-64 years), thus suggesting that infections are taking place early in life. Globally, around $20 \%$ of all cervical cancers are being diagnosed between the ages of $15-39$ years. $^{8}$ This suggests that a proportion of infections may be occurring even earlier.

Previous reports have shown a high prevalence of HPV infections in younger populations ${ }^{9} 10$ and reports in southern Africa demonstrated a high prevalence of HPV infections among women of 30 years or younger. ${ }^{11} 12$ Limited information is available about the HPV epidemiology in Mozambique particularly in men and young populations in urban settings. Castellsagué et al have shown that the HPV prevalence is high $(75 \%)$ in women aged 14-61 years in rural southern Mozambique. ${ }^{13}$ Cervical cancer is the most frequent cancer in Mozambican women. ${ }^{14}$ Others have described the contribution of HPV infection in the development of cervical cancers in Mozambique. ${ }^{15-18}$

By the end of 2016, HPV vaccination had only been introduced in 65 countries worldwide, seven being in the African continent. In Mozambique, HPV vaccine has yet not been introduced in the national expanded programme on immunisation. Currently, 23 African countries, including Mozambique, are conducting or have recently finalised their HPV vaccine pilot projects. ${ }^{1}$

The following three HPV vaccines are available: Gardasil (which confers protection against HPV6, $-11,-16$ and -18 and cross-protection against HPV31); Cervarix (which prevents against HPV16 and -18 and provides high and moderate cross-protection against HPV31, -45 and -33 , respectively); and Gardasil 9 (which protects against HPV6, $-11,-16,-18,-31,-33,-45,-52$ and -58$)$. Gardasil 9 is a second-generation 9-valent vaccine that can theoretically prevent $87 \%$ of all hr-HPV infections in Africa. ${ }^{19}$ Nonetheless, the HPV distribution varies between African regions, and the identification of country-specific profiles and their adequacy to the currently available vaccines remains of great importance when deciding on vaccine introduction.
In the present study, we aimed to (1) determine the prevalence and distribution of HPV infections in sexually active young adults and (2) determine the suitability of the current HPV vaccines in the context of the Mozambican epidemic.

\section{SUBJECTS AND METHODS}

This report adheres to the Strengthening The Reporting of OBservational Studies in Epidemiology (STROBE) guidelines for the reporting of observational studies.

\section{Study design and population}

This was a cross-sectional ancillary study of an HIV incidence study conducted at a youth clinic in Maputo city, Mozambique, from August 2009 to October $2011 .{ }^{20}$ In the parent study, briefly, 1380 males and females aged 18-24 years were screened for HIV, syphilis and hepatitis B virus. HIV-seronegative subjects were enrolled and followed for 1 year with quarterly visits to the clinic for assessment of HIV serostatus. Baseline demographic and behavioural data were collected using structured questionnaires. In the present study, samples from male and female subjects were collected at one time point, at screening or at one of the follow-up visits. We aimed at sampling 500 individuals with equal gender distribution (250 females and 250 males) based on the possibility of recruiting male subjects at the youth clinic, which mainly caters to women. Cervical samples were collected in female participants via speculoscopy, using a Rovers Viba-Brush (Rovers Medical Devices BV, Oss, the Netherlands). Urethral samples were collected from male participants by gently inserting a cotton swab approximately $2-4 \mathrm{~cm}$ in the urethral meatus and rotating it in one direction. Both the brushes and swabs were immersed in $5 \mathrm{~mL}$ of SurePath cell-preservation solution (TriPath Imaging, Burlington, North Carolina, USA), transported to the study laboratory within the same day of collection and stored at $+4^{\circ} \mathrm{C}$ to $+8^{\circ} \mathrm{C}$ for 3 months and then at $-80^{\circ} \mathrm{C}$.

\section{Laboratory testing}

DNA was extracted from an initial sample volume of $1.5 \mathrm{~mL}$ using a QIAamp DNA Mini Kit (Qiagen, GmbH, Hilden, Germany) according to the manufacturer's instructions and eluted to a final volume of $200 \mu \mathrm{l}$.

HPV genotyping was performed using the CLART Human Papillomavirus 2 (Genomica, Madrid, Spain), a low-density microarray platform based on PCR amplification of a $450 \mathrm{bp}$ fragment within an HPV L1 highly conserved region from 35 different HPV types (HPV6, $-11,-16,-18,-26,-31,-33,-35,-39,-40,-42,-43$, $-44,-45,-51,-52,-53,-54,-56,-58,-59,-61,-62,-66$, $-68,-70,-71,-72,-73,-81,-82,-83,-84,-85$, and -89$)$ and a human gene control (CTFR). Individual genotyping results were analysed on the Clinical Array Reader (Genomica, Madrid, Spain). Adequacy of samples was assessed by amplification of the CTFR. Samples with undetectable DNA were rerun and the second result was considered final. The CLART Human Papillomavirus 2 
assay complies with the European Union (EU) safety, health or environmental requirements, with the EU legislation and with the European In-Vitro Diagnostic Devices Directive. This assay has been shown to have a similar performance as that of other well-established HPV screening assays, such as the Food and Drug Administration-approved Hybrid Capture 2 test. $^{21} 22$

DNA extraction was performed at the Instituto Nacional de Saúde laboratories in Maputo, Mozambique. DNA samples were then shipped to the Molecular Biology laboratories at the Department of Laboratory Medicine, Faculty of Medicine and Health, Örebro University Hospital, Örebro, Sweden for HPV genotyping.

HIV testing was performed as previously described on site in Maputo. ${ }^{20}$

\section{Statistical analysis}

Demographic and risk behavioural data were entered into a MySQL database V.5.1 (MySQL AB, 2008) and HPV laboratory results in a Microsoft Office Excel 2010 spreadsheet. For processing and analysis, data were imported into Stata V.14 (StataCorp. 2015. Stata: Release 14. Statistical Software. College Station, TX: StataCorp LP).

All analyses were stratified by gender. Descriptive statistics were employed as follows: frequencies for categorical variables, and means and SD for quantitative variables. We used list-wise deletion as the missing values were negligible. Here, we report the prevalence of HPV infections and HPV genotypes per gender and HIV status. Bivariate logistic analysis between sociodemographic and sexual behavioural characteristics and presence of any HPV infection was conducted. Age of individuals and age of sexual debut and characteristics whose $p$ value on bivariate logistic regression analysis was below 0.25 were included in the multivariable logistic regression. Additionally, to identify characteristics that would contribute to multiple HPV genotype infections a multinomial logistic regression (MNLR) was used. For this analysis, males and females are not stratified given the sample constraints. We first grouped the participants as follows: (a) subjects without an HPV infection; (b) subjects with one HPV infection (monoinfection); and (c) subjects with at least two HPV infections (multiple infections). The MNLR per level of the factor under consideration provided two ORs (one for monoinfected versus not-infected subjects and another for the subjects with multiple-infections versus not-infected subjects). We report both the unadjusted and adjusted ORs and their respective 95\% CI. For adjustment, we included factors whose $p$ values through the global likelihood ratio test were below 0.25 in the MNLR bivariate analysis; gender and age were forced factors to include in the model. The significance level was set at $5 \%$.

\section{Ethics statement}

Ethical approval was granted by the National Health Bioethics Committee of Mozambique (Ref. 148/CNBS of 8 May 2009 and Ref. 18/CNBS/11). Testing performed in Sweden was approved by the Regional Ethical Review
Board Uppsala. Study investigators followed the Good Clinical Practice-International Conference on Harmonisation (GCP-ICH) guidelines. Subjects signed an informed consent form prior to any study activities.

\section{RESULTS}

In total, 489 participants (263 females and 226 males) were enrolled, but samples from $77(15.7 \%)$ subjects had undetectable DNA levels and were excluded from the data analysis, being $27 / 263(10.3 \%)$ from females and $50 / 226(22.1 \%)$ from males.

\section{Demographic and behavioural characteristics}

Table 1 shows the socio demographic and sexual behavioural characteristics of study participants by sex. Of the 412 subjects with valid HPV results, 236 (57.3\%) were females, and $176(42.7 \%)$ were males. The mean age of study participants was 21.1 years (SD: 1.71) with males being slightly older than females (21.5 vs 20.8). Almost all participants were students $(94.2 \%)$ and all had some formal education. Male subjects were more educated than females with more than half having secondary or higher educational degrees (56.8\% vs $41.9 \%)$. Nearly all participants were single $(98.8 \%)$. The median age at sexual debut was 17 years (IQR: 15-18) being 16 years (IQR: 14-18) for males and 17 years (IQR: 16-18) for females. The majority of subjects $(89.3 \%)$ had more than one sexual partner in life $(97.2 \%$ of males and $83.5 \%$ of females) and $24 \%$ reported two or more sexual partners in the last 6 months $(37.5 \%$ of males and $14 \%$ of females). Around one-fourth of the study population reported having at least one episode of STI in life and this was seen more frequently in women than in men $(34.3 \%$ vs $13.6 \%$ ). Only $66.0 \%$ of subjects reported having used a condom at the time of their last sexual intercourse with females reporting a lower frequency compared with males (59.7\% vs $74.4 \%)$. HIV infection was diagnosed in 21 $(5.1 \%)$ participants, of which $17(81.0 \%)$ were females.

\section{HPV prevalence and genotype distributions}

The overall HPV prevalence was 40.8\% (168 infected subjects; $95 \%$ CI 36.0 to 45.5$)$ and was significantly higher in women than in men $(150 / 236$ [63.6\%, 95\% CI 57.1 to 69.7 ] vs $18 / 176$ [10.2\%, $95 \%$ CI 6.2 to 15.7$], \mathrm{p}<0.001)$. The HPV prevalence among HIV-infected subjects (14/21, $66.7 \%$ ) was $69 \%$ higher than in the HIV-negative subjects $(154 / 391,39.4 \%$; $\mathrm{p}=0.013)$. Figure 1 and table 2 show the distribution of HPV genotypes in male and female participants. Of the 412 enrolled participants, 115 (27.9\%), 54 $(13.1 \%)$ and $89(21.6 \%)$ were infected with one or more high-risk, probably high-risk and low-risk HPV types, respectively. High-risk and phr-HPV types were present in $124 / 150(82.7 \%, 95 \%$ CI 75.6 to 88.4$)$ and $14 / 18$ (77.8\%, 95\% CI 52.4 to 93.6) of HPV-infected women and men, respectively $(\mathrm{p}=0.533)$. Overall, HPV52 was the most frequent type found $(9.1 \%)$, followed by HPV $35,-6,-16$, $-53,-58$ and -51 . Altogether, these HPV types accounted for $49.9 \%$ of the infections in women and men. In female 
Open Access

Table 1 Sociodemographic and sexual behavioural characteristics of study participants by sex

\begin{tabular}{|c|c|c|c|c|c|c|}
\hline & Total & & Male & & Female & \\
\hline & $\mathbf{n}$ & $\%$ & n & $\%$ & n & $\%$ \\
\hline Total & 412 & 100.0 & 176 & 100.0 & 236 & 100.0 \\
\hline \multicolumn{7}{|l|}{ Age (years) } \\
\hline Mean (SD) & \multicolumn{2}{|l|}{$21.1(1.71)$} & \multicolumn{2}{|l|}{$21.5(1.67)$} & \multicolumn{2}{|l|}{$20.8(1.68)$} \\
\hline \multicolumn{7}{|l|}{ Marital status } \\
\hline Single & 407 & 98.8 & 175 & 99.4 & 232 & 98.3 \\
\hline Married/cohabitating & 5 & 1.2 & 1 & 0.6 & 4 & 1.7 \\
\hline \multicolumn{7}{|l|}{ Education } \\
\hline Secondary or less & 213 & 51.7 & 76 & 43.2 & 137 & 58.1 \\
\hline Technical training & 109 & 26.5 & 50 & 28.4 & 59 & 25.0 \\
\hline University grade & 90 & 21.8 & 50 & 28.4 & 40 & 16.9 \\
\hline \multicolumn{7}{|l|}{ Occupation } \\
\hline Student & 388 & 94.2 & 163 & 92.6 & 225 & 95.3 \\
\hline Employed & 23 & 5.6 & 12 & 6.8 & 11 & 4.7 \\
\hline Missing & 1 & 0.2 & 1 & 0.6 & 0 & 0.0 \\
\hline \multicolumn{7}{|l|}{ Religion } \\
\hline Christian & 375 & 91.0 & 162 & 92.0 & 213 & 90.3 \\
\hline Other & 37 & 9.0 & 14 & 8.0 & 23 & 9.7 \\
\hline \multicolumn{7}{|l|}{ Age at sexual debut } \\
\hline Less than 18 & 285 & 69.2 & 115 & 65.3 & 170 & 72.0 \\
\hline 18 or more & 122 & 29.6 & 58 & 33.0 & 64 & 27.1 \\
\hline Missing & 5 & 1.2 & 3 & 1.7 & 2 & 0.8 \\
\hline \multicolumn{7}{|c|}{ Number of sex partners in life } \\
\hline $0-1$ & 44 & 10.7 & 5 & 2.8 & 39 & 16.5 \\
\hline$>1$ & 368 & 89.3 & 171 & 97.2 & 197 & 83.5 \\
\hline \multicolumn{7}{|c|}{$\begin{array}{l}\text { Number of sex partners in the last } \\
6 \text { months }\end{array}$} \\
\hline $0-1$ & 313 & 76.0 & 110 & 62.5 & 203 & 86.0 \\
\hline$>1$ & 99 & 24.0 & 66 & 37.5 & 33 & 14.0 \\
\hline \multicolumn{7}{|c|}{$\begin{array}{l}\text { Condom use in the last sexual } \\
\text { intercourse }\end{array}$} \\
\hline No & 139 & 33.7 & 45 & 25.6 & 94 & 39.8 \\
\hline Yes & 272 & 66.0 & 131 & 74.4 & 141 & 59.7 \\
\hline Missing & 1 & 0.2 & 0 & 0.0 & 1 & 0.4 \\
\hline \multicolumn{7}{|l|}{ Had a STI in life } \\
\hline No & 305 & 74.0 & 150 & 85.2 & 155 & 65.7 \\
\hline Yes & 105 & 25.5 & 24 & 13.6 & 81 & 34.3 \\
\hline Missing & 2 & 0.5 & 2 & 1.1 & 0 & 0.0 \\
\hline \multicolumn{7}{|l|}{ HIV infection } \\
\hline Positive & 21 & 5.1 & 4 & 2.3 & 17 & 7.2 \\
\hline Negative & 391 & 94.9 & 172 & 97.7 & 219 & 92.8 \\
\hline
\end{tabular}

STI, sexually transmitted infection.

participants, the most common genotypes were similar to the overall distribution (HPV52, $-35,-16,-53,-58,-6$, and -51$)$ and accounted for half $(50.3 \%)$ of the infections in women (figure 1A). In contrast, male participants'
HPV infections were less diverse with HPV51 being the most frequent type found, followed by HPV6, $-11,-52$, -59 and -70 in equal proportions. These six types were responsible for $52 \%$ of the infections in men (figure 1B). 


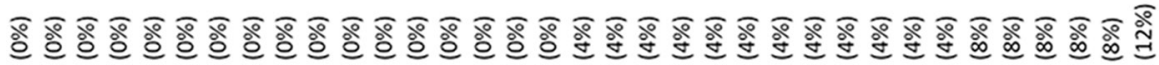

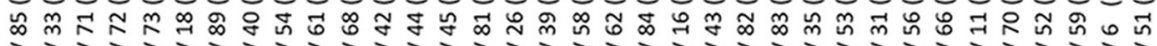

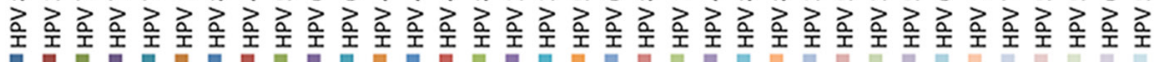
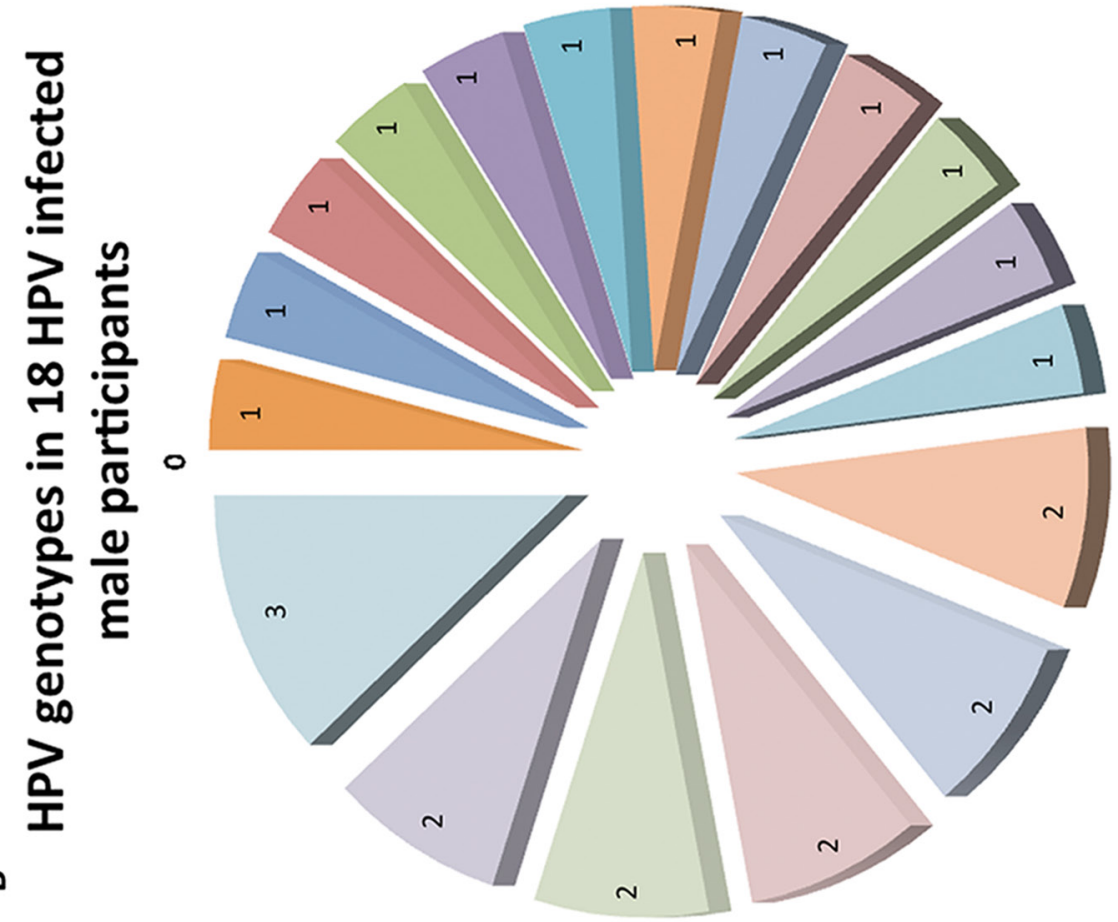

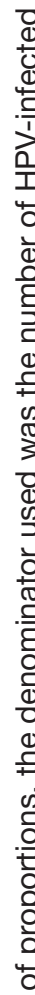

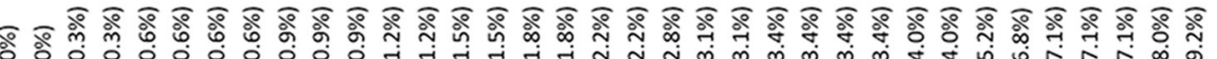

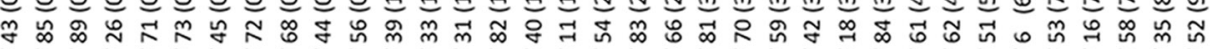

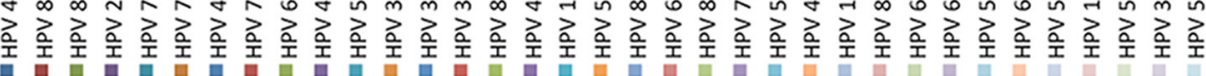

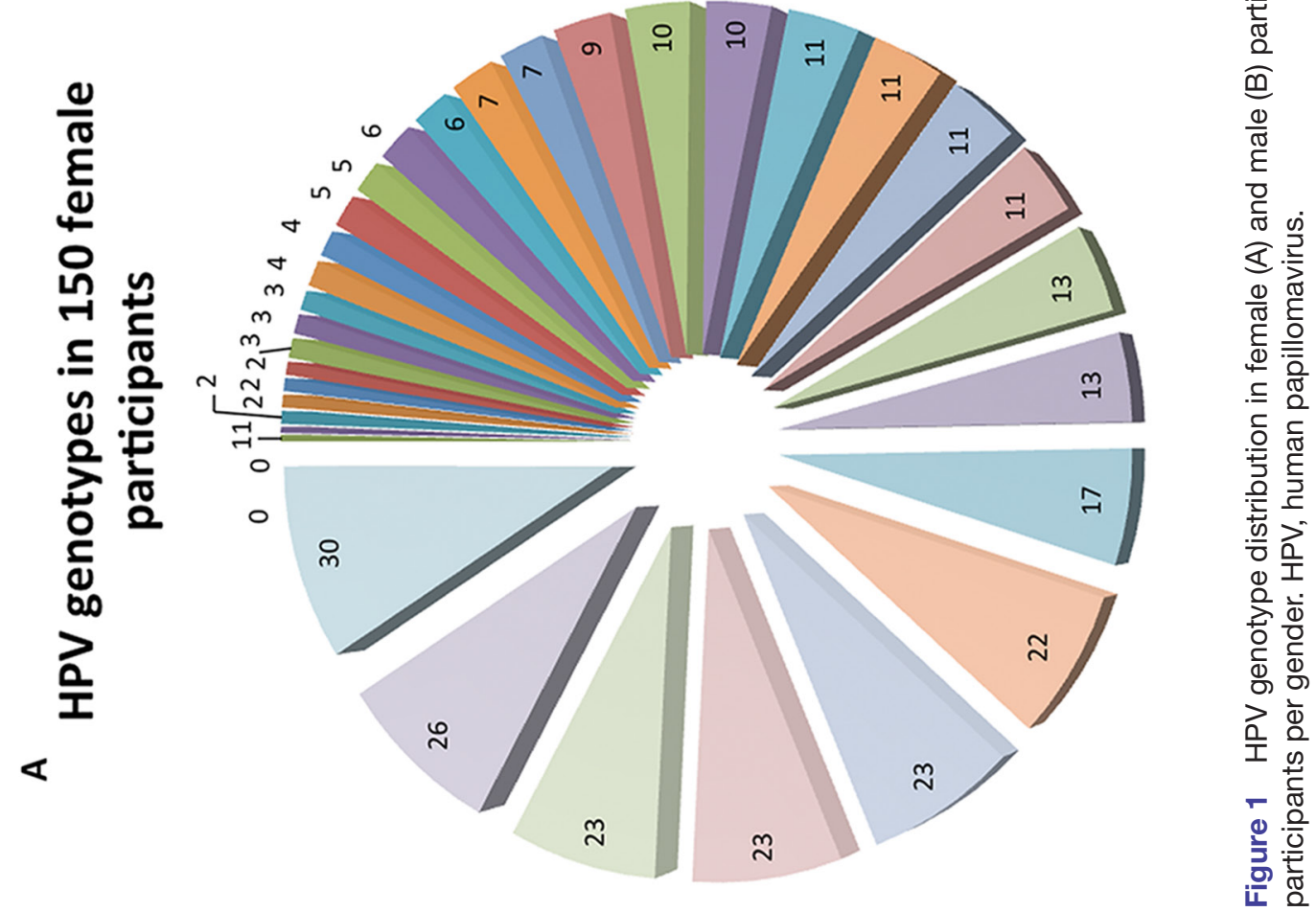




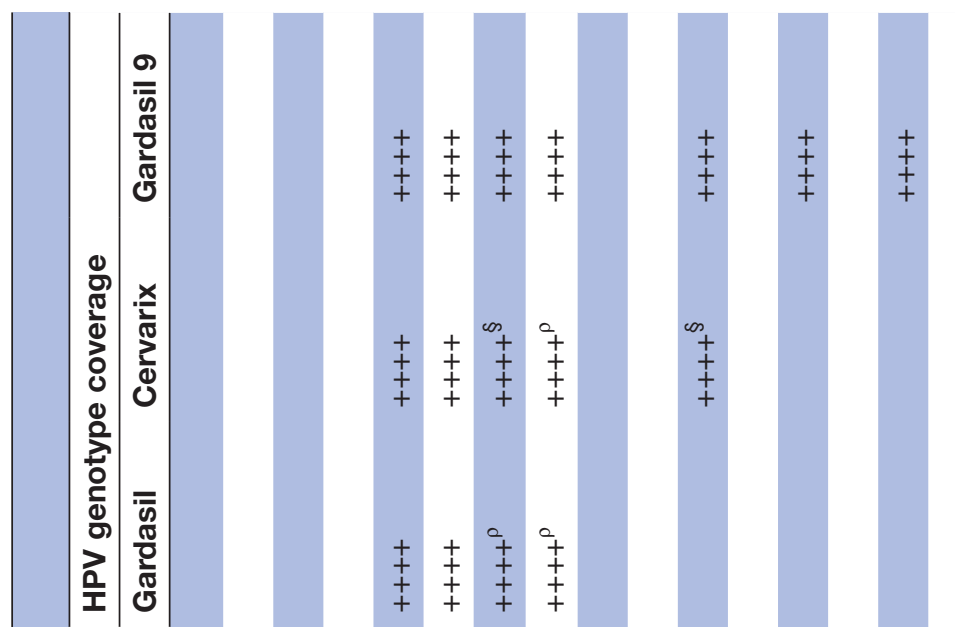

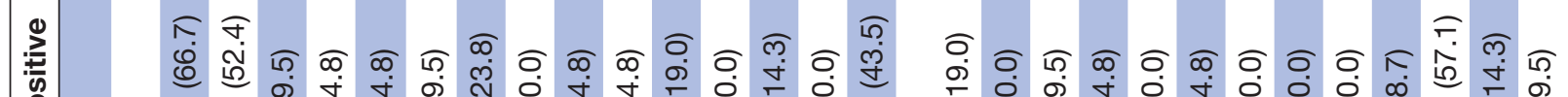

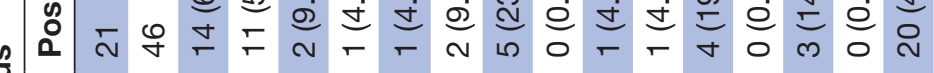

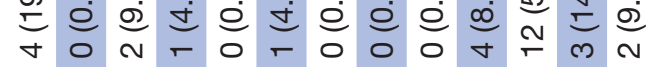

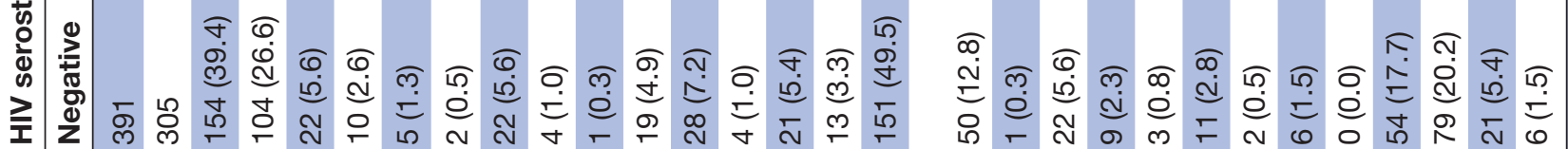

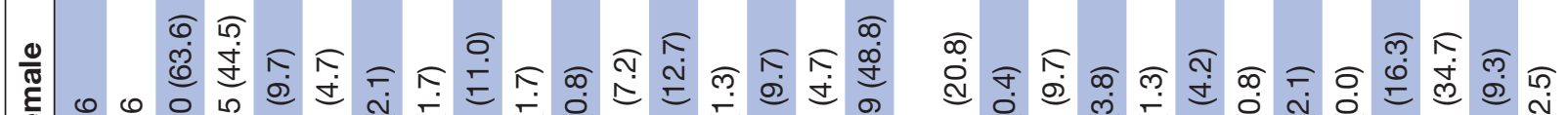

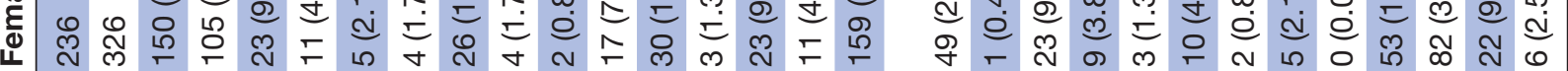

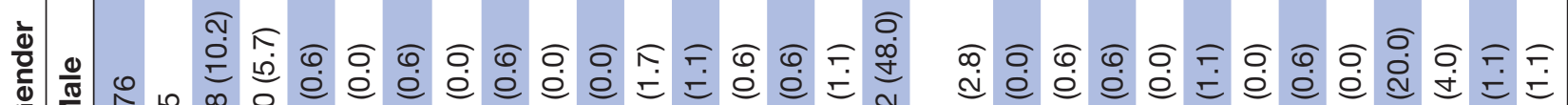

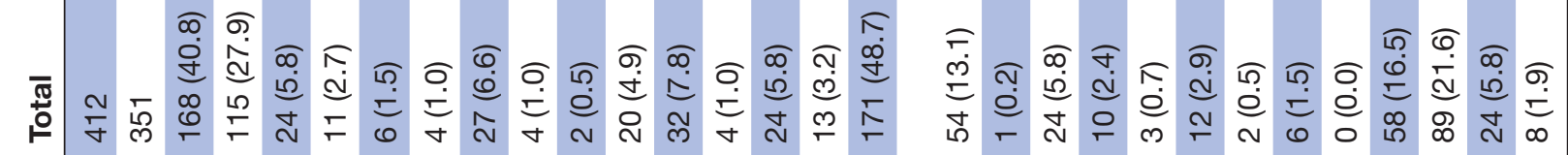

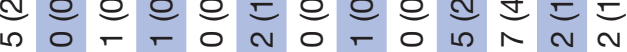

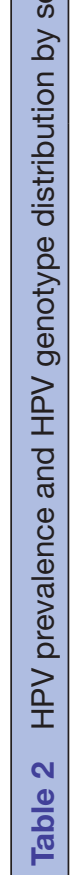

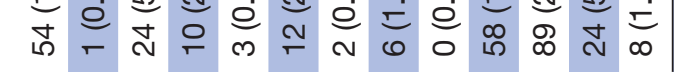
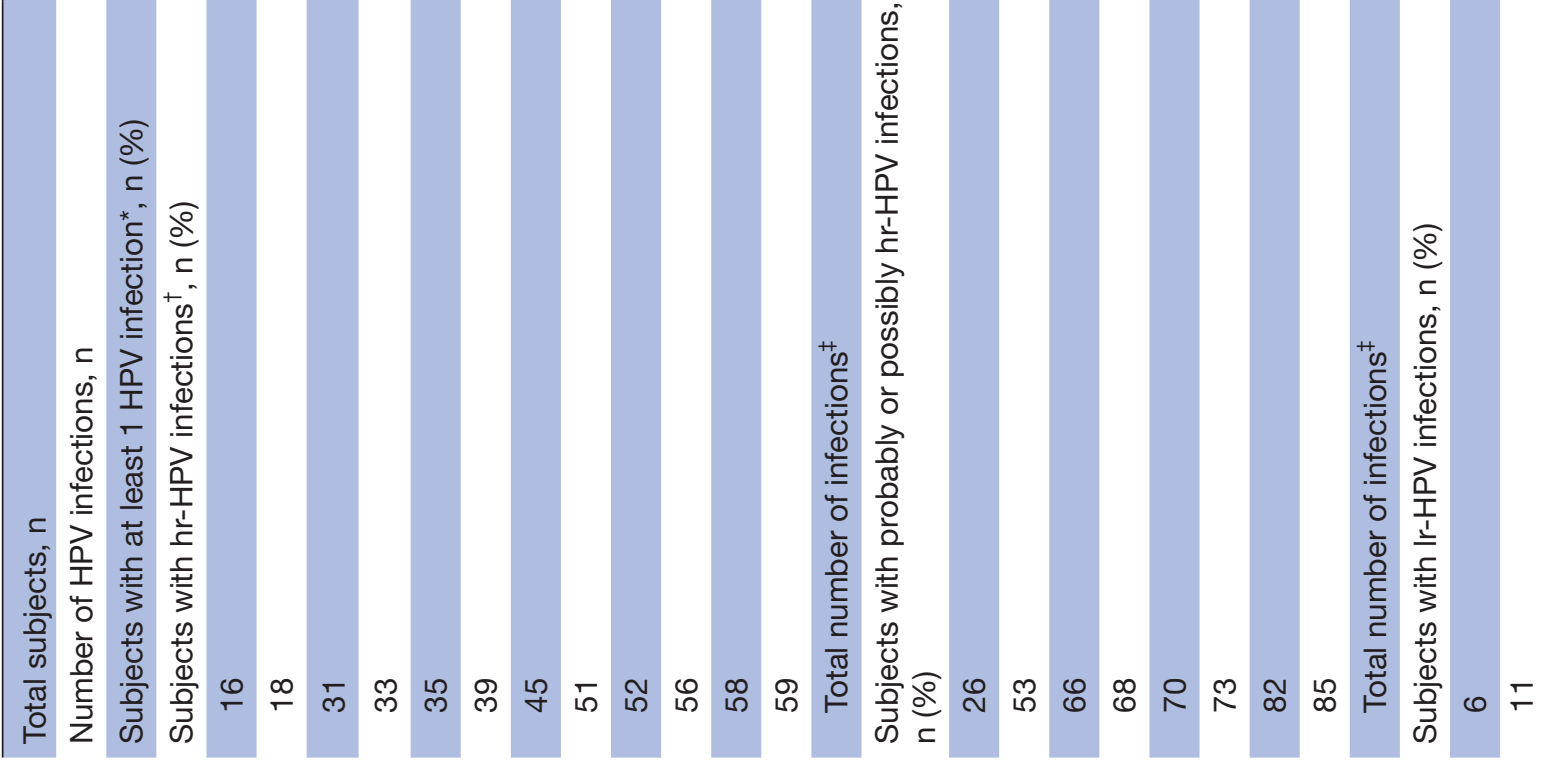


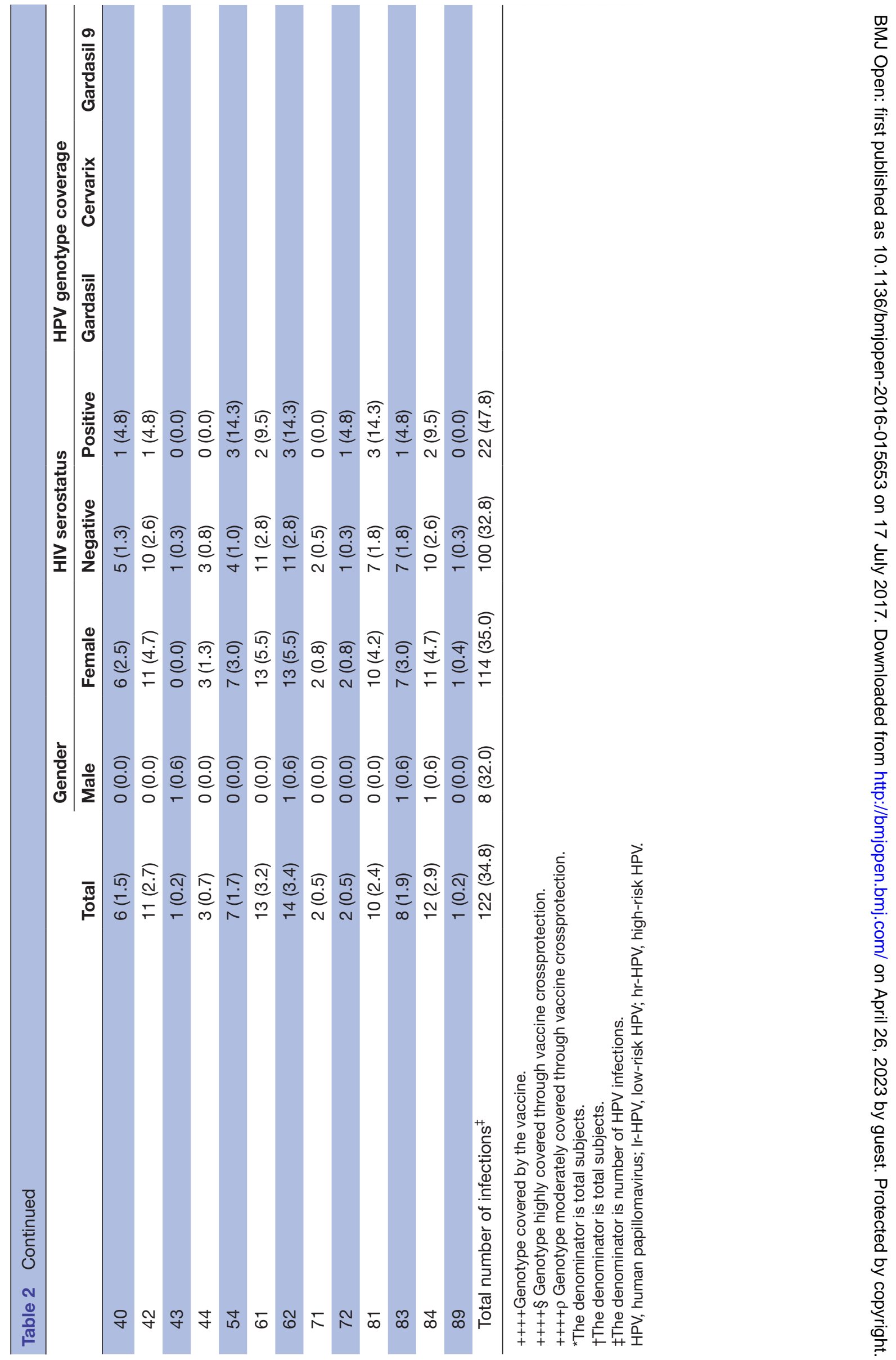




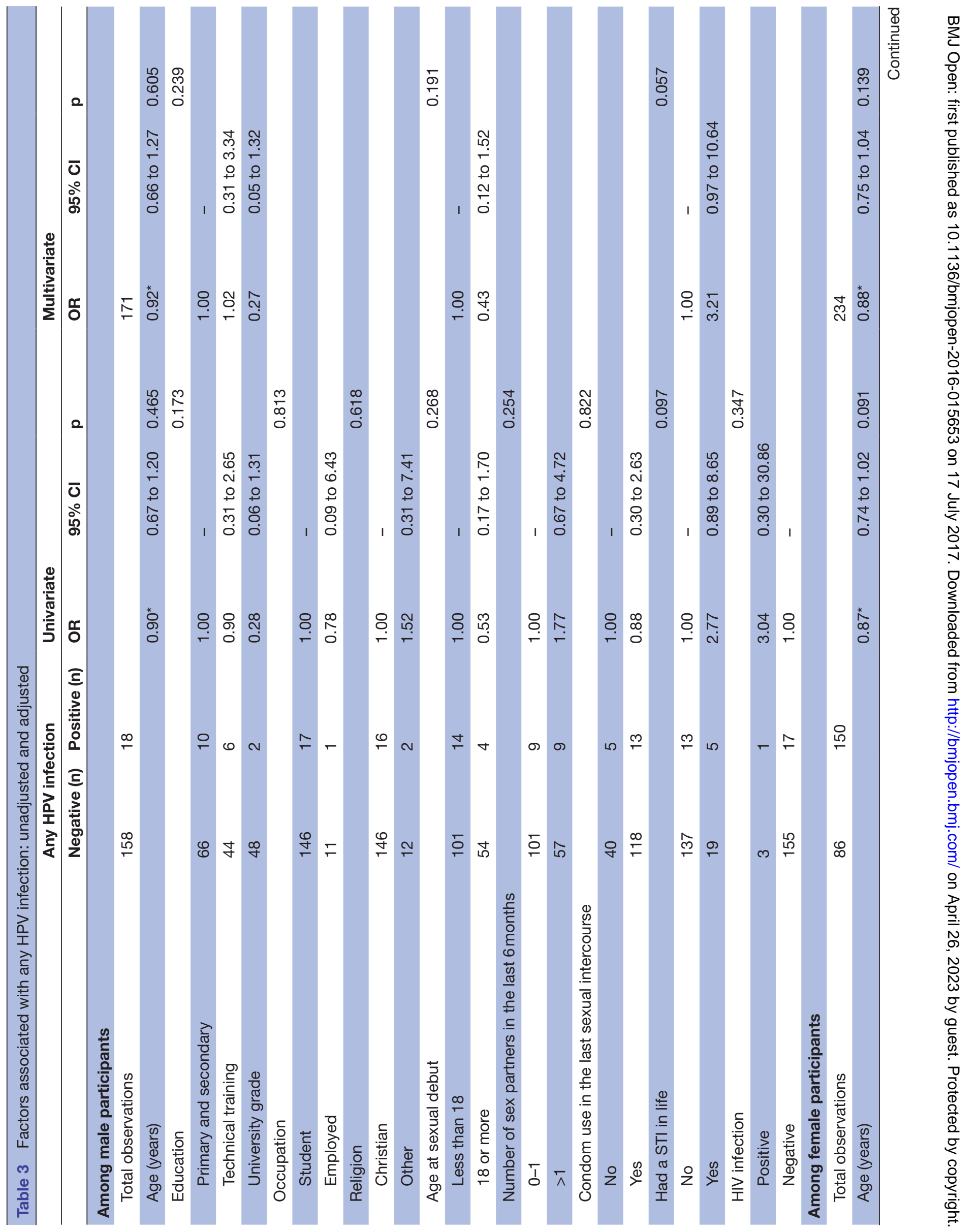




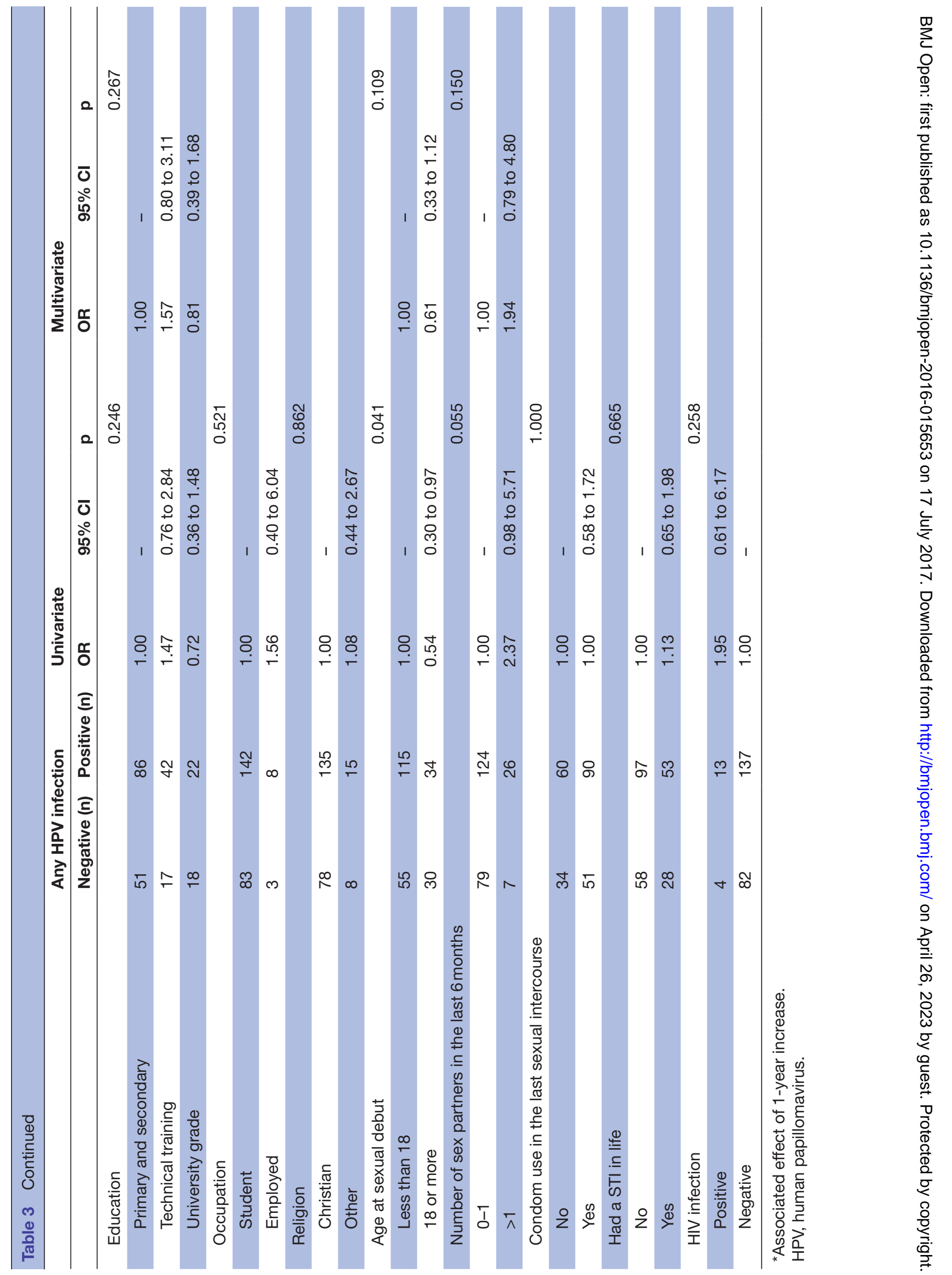


Hr-HPV genotypes were identified in $11 / 21(52.4 \%)$ of HIV-infected subjects. Three hr-HPVs (HPV35, -52 and -58 ) accounted for $60 \%$ of all hr-HPV infections in this population. There were no differences in the number of subjects infected with hr-HPVs and phr-HPVs in the HIV-infected $(\mathrm{p}=0.552)$ and uninfected populations $(\mathrm{p}=1.000)$. In contrast, lr-HPVs were more frequently observed in the HIV-infected subjects $(\mathrm{p}=0.022)$.

\section{Vaccine-matched HPV genotypes}

Table 2 shows the distribution of HPV genotypes in women and men, and their correspondence to the genotypes present in the current HPV vaccines. Genotypes associated with vaccine crossprotection were included in the analysis and considered as covered by the vaccine. The total number of circulating HPV genotypes matching the vaccine genotypes were 5/33 (15.2\%; Gardasil: HPV6,-11, $-16,-18$ and -31 ) , 5/33 (15.2\%; Cervarix: HPV16, -18 , $-31,-33$ and -45$)$ and 9/33 (27.3\%; Gardasil 9: HPV6, $-11,-16,-18,-31,-33,-45,-52$ and -58$)$ for the female population and 4/18 (22.2\%; Gardasil: HPV6, $-11,-16$ and -31$), 2 / 18$ (11.1\%; Cervarix: HPV6 and -31 ) and 6/18 (33.3\%; Gardasil 9: HPV6, $-11,-16,-31,-52$ and $-58)$ for the male population. The three vaccines can cover 3/19 (15.8\%; Gardasil: HPV16, -18 and -31$)$, 5/19 (26.3\%; Cervarix: HPV16, $-18,-31,-33$ and -45$)$ and 7/19 (36.8\%; Gardasil 9: HPV16, -18, -31, -33, -45, -52 and -58$) \mathrm{hr}-H P V$ s circulating in women and 2/12 (16.7\%; Gardasil: HPV16 and -31), 2/12 (16.7\%; Cervarix: HPV16 and -31) and 4/12 (33.3\%; Gardasil 9: HPV16, -31, -52 and -58 ) in men.

\section{Factors associated with HPV infection}

Overall, women had a significantly higher HPV infection prevalence than men $(p<0.001)$. Although the odds of being infected with HPV reduced by $80 \%$ for each year of age, in the multivariate analysis this was not significant. Sexual debut before the age of 18 years, history of STI and infection with HIV were significantly associated with the presence of HPV infection ( $\mathrm{p}=0.008, \mathrm{p}<0.001, \mathrm{p}=0.013$, respectively) in the univariate analysis. When stratifying by gender (table $3 \mathrm{~A}, \mathrm{~B})$, the univariate analysis show that women who initiated sexual activity before the age of 18 were significantly more at risk of having a HPV infection $(\mathrm{p}=0.041)$, and a marginally significance association was seen in women who reported to have had more than two sexual partners in the last 6 months $(\mathrm{p}=0.055)$, but this was not seen in the multivariate analysis. No significant associations were seen for male participants both in the univariate and multivariate analysis, but a marginally significant association was seen for a reported history of STI and HPV infection.

\section{HPV monoinfection versus multiple infections}

Almost half, 75/168 (44.6\%), of HPV infected subjects had a single infection, 48/168 (28.6\%) had two concurrent HPV infections and 45/168 (26.8\%) had three or more HPV coinfections (figure 2). HPV-infected male

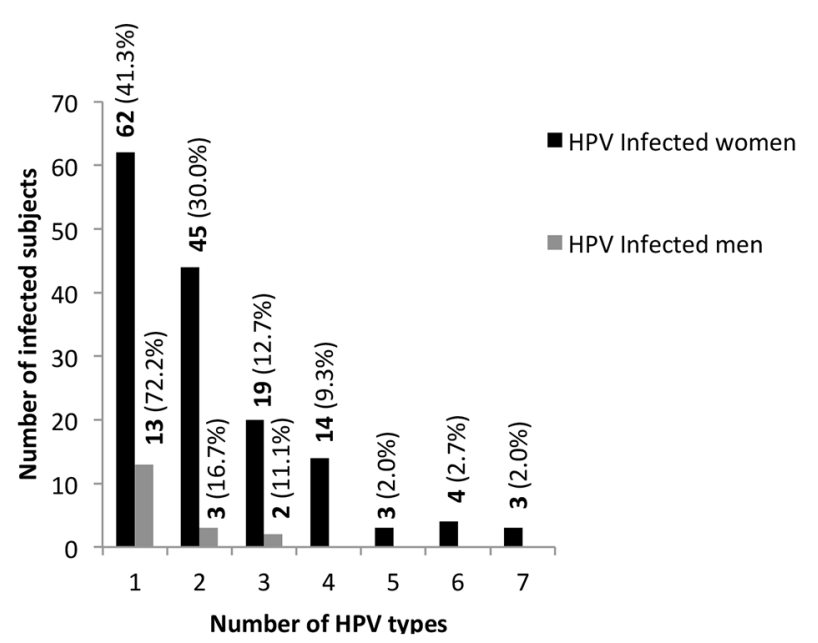

Figure 2 Distribution of HPV infections in female and male participants. The denominator is the total number of infected women and men, respectively. HPV, human papillomavirus.

participants had more frequently a single infection (72.2\% of the cases), whereas more than $50 \%$ of HPV-infected females had two or more HPV coinfections. In the univariate analysis, several factors were shown to be associated with the presence of two or more (multiple) HPV coinfections. Being a woman, younger age (the odds of being infected with HPV reduced by $80 \%$ for each year of age), lower educational degree, sexual debut before the age of 18, reported STI in life and the presence of a HIV infection were factors associated with multiple-HPV infections. Nonetheless, in the multivariate analysis only three factors were demonstrated to be significantly associated, namely, being a woman $(\mathrm{p}=0.001)$, having started sexual activity before the age of $18(\mathrm{p}=0.008)$ and the presence of a HIV infection $(p=0.003)$. Condom use in the last sexual intercourse was not significantly associated with protection against HPV infection $(\mathrm{p}=0.143)$ (table 4).

\section{Discussion}

This was a cross-sectional study reporting the prevalence of HPV infections and genotype distributions in young adults aged 18-24 in Maputo city, Mozambique. The HPV prevalence in the female population was $63.6 \%$ (95\% CI 57.1 to 69.7 ) and was similar to previous reports of $75.9 \%$ in women aged 14-61 years in rural southern Mozambique and other neighbouring countries. ${ }^{11-1323}$ The HPV prevalence in the male population was $10.2 \%$ (95\% CI 6.1 to 15.7$)$, which was lower than previous reports for African countries and other regions. ${ }^{2}$ Although no significant difference was observed in the number of hr-HPV infections between women and men, a high susceptibility of women to HPV infections was demonstrated.

We report a lower prevalence of HPV infections in men than in women, which is contrary to what has been shown in neighbouring countries and other regions. ${ }^{24}$ Studies assessing different male anatomic collection sites show that HPV detection in urethral samples is suboptimal compared with other sampling sites in the male genitalia, 


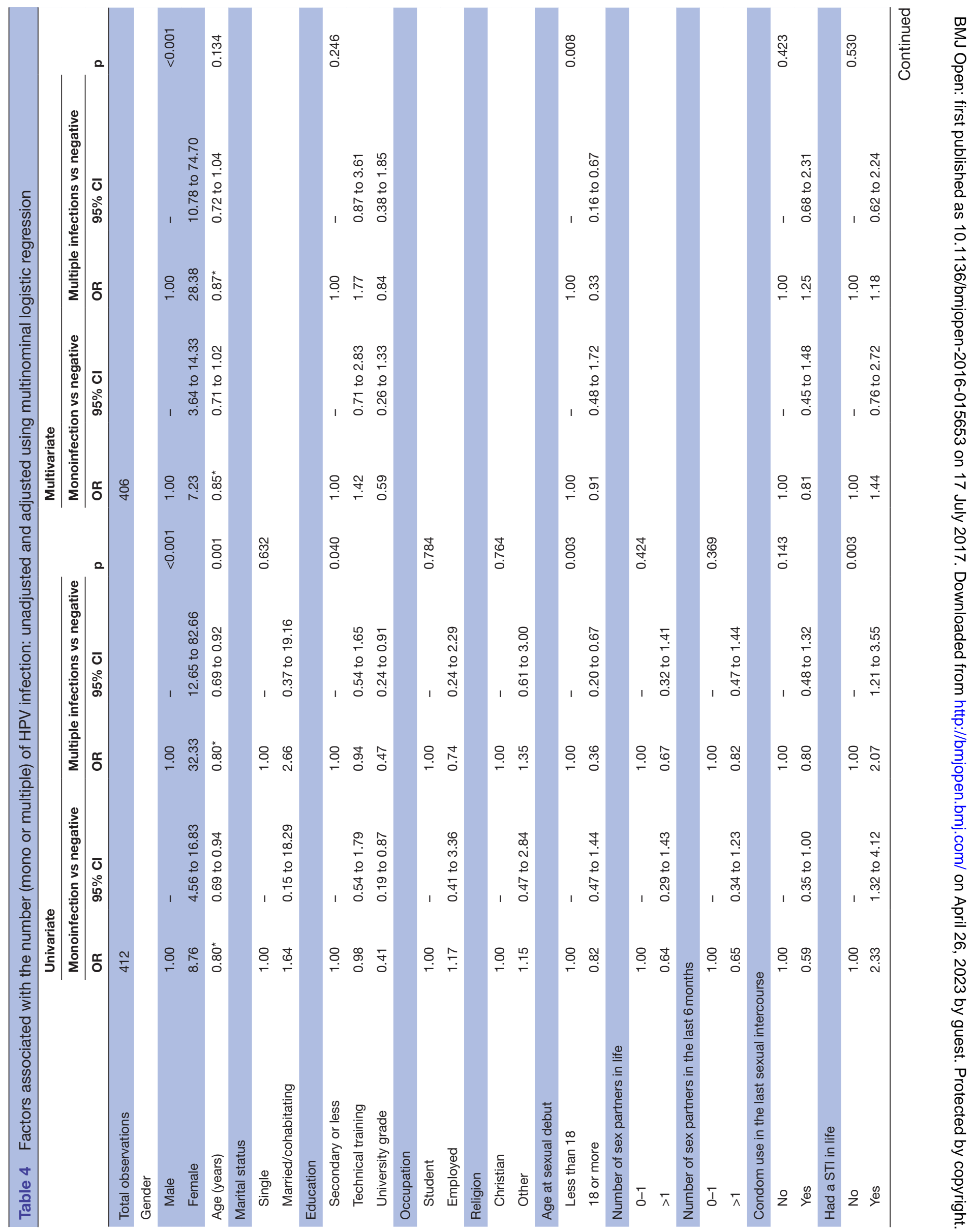


but the sensitivity reported for urethral sampling has varied. ${ }^{25}{ }^{26}$ In a study comparing sampling from seven male genital sites, Giuliano et al reported that detection was highest at the penile shaft $(48 \%)$, while only $10 \%$ of HPV infections were detected when using urethral sampling. ${ }^{26}$ In contrast, the sensitivity of urethral samples for overall HPV detection was $54.2 \%$ in a study that explored expressed prostate secretions as an option for detection of HPV infections in Russian men. ${ }^{27}$ We found $50 / 226(22.1 \%)$ urethral samples with undetectable DNA and $158 / 176(89.8 \%)$ of valid samples tested negative. The first could be related to an inadequate sampling technique and the second to the selected anatomic collection site. Altogether, this could have contributed to the lower male HPV prevalence found in our study.

Worldwide, the prevalence of cervical HPV infections in healthy women has been demonstrated to be $11.7 \%$. Nonetheless, in less developed regions, such as East Africa, the prevalence is three times the global figures $(33.6 \%) .^{28}$ High-risk HPV16, - 18, -52, $-31,-58,-39$, -51 and -56 have been found in women with normal cytology. In this study, we demonstrated that hr-HPV genotype distribution in Mozambican healthy young women differs from the global figures with HPV52, -35 , $-16,-53,-58$ and -51 being the most prevalent. Castellsagué et al have previously described that hr-HPVs 51, $-35,-18,-31$ and -52 were the most commonly found in women aged 14-61 years with normal cytology in a study conducted in southern Mozambique, ${ }^{13}$ which is similar to our findings. Furthermore, HPV16 contributed to only $6.8 \%$ of female infections in the present study. These findings are concordant with those from Castellsague $e t$ $a l .{ }^{13}$ Globally, HPV16 has been responsible for $22.5 \%$ of all infections in women, whereas in sub-Saharan Africa, it seems to have the lowest contribution $(13.7 \%, 11.3 \%$ and $11.1 \%$ for Southern, Eastern and Western Africa, respectively). ${ }^{28}$ The peak prevalence of HPV infections in women was shown to occur in early ages (before the age of 30 years).$^{11}{ }^{12}$ Some reports have also described a less pronounced second peak prevalence in older women (around their 50s). Reasons for the occurrence of the first HPV peak are related to absence of immune responses to HPV and sexual activity in younger women. The second peak is not consistently reported across the globe, but it is speculated that hormonal changes my play a role. Although the majority of HPV infections in early ages clear spontaneously, ${ }^{29}{ }^{30}$ some do become chronic infections and may potentially evolve to malignant transformation of the cervix. In the present study, we described the epidemiology of HPV infections in both women and men that belong to the 'first HPV peak' population.

Our report shows that more than $50 \%$ of the study population had two or more concomitant HPV infections with more than $50 \%$ of female participants having two or more coinfections compared with only $27.8 \%$ of male participants. It has been demonstrated by others that women with multiple HPV infections take longer to clear their infections compared with women with a 
single infection, ${ }^{29}{ }^{30}$ thus potentially contributing to the establishment of a persistent infection, a prerequisite for cervical cancer development. Several studies conducted in Mozambique have shown a strong association between HPV16, $-33,-35,-45,-18,-31$ and -58 with cervical neoplasia. ${ }^{15-18}$ In another Mozambican study, HPV52 was reported to be the second most common hr-HPV in women with abnormal cytology and the fourth most common in women with invasive cervical cancer. ${ }^{13}$ In the present study, we demonstrated a 50\% match between the hr-HPV types commonly circulating in the female population (HPV35, -16, -52 and -58) and HPVs types commonly present in cervical neoplasia in Maputo, Mozambique.

We confirmed the association between early onset of sexual debut, history of past STI and HIV infection with multiple HPV infections. ${ }^{31}{ }^{32}$ These findings reiterate the need to intensify sexual behaviour education and STI prevention in young populations.

This study shows a high prevalence of HPV infections $(66.7 \%)$ in the HIV-infected population. A previous report showed a prevalence of HPV infections of 56.6\% in HIV-infected African women, which is concordant with our findings. ${ }^{33}$ Other reports from South Africa have demonstrated an even higher HPV prevalence $(75 \%)$ in the age group of $17-19$ years. ${ }^{34}$ We demonstrated that HPV infections and multiple HPV infections are more frequent in HIV-infected subjects. Similar descriptions have been previously reported. ${ }^{34}$ We have shown that HPV 35, -52 and -58 were responsible for $60 \%$ of all hr-HPV infections in this population. McDonald et al have reported that hrHPV35, $-58,-18,-45,-16$ and -52 were the most common genotypes in HIV-infected women with normal cytology in neighbouring South Africa. ${ }^{34}$ Lastly, other reports in Africa have shown that the most frequent genotypes circulating in HIV-infected women were HPV16, $-58,-52,-31,-35$ and $-18 .^{33}$ These findings suggest a high homology between the common hr-HPV genotypes in Mozambique versus South Africa and other African regions. We have also described a high prevalence of lr-HPV infections in the HIV-infected subjects. These findings were also demonstrated elsewhere. ${ }^{36}$

Since 2014, Mozambique has been engaged in the HPV vaccine demonstration project which aimed at accessing the country's preparedness for the introduction of an $\mathrm{HPV}$ vaccine in the national vaccination programme. Information regarding HPV types circulating in young populations is crucial for selection of a suitable vaccine. Currently available HPV vaccines, Gardasil, Cervarix and Gardasil 9 can prevent up to seven hr-HPV infections. Moderate to high crossprotection against HPV types phylogenetically related to HPV16 and -18 have also been reported with the use of Gardasil and Cervarix. Nonetheless, the level of protection has shown to be heterogeneous across the studies and a signal for reduction of vaccine efficacy with extended follow-up has also been demonstrated. ${ }^{37}$ Our study shows that Gardasil 9 has the highest genotype coverage in both Mozambican women and men, whereas Gardasil has the lowest in women and Cervarix in men. Gardasil 9 can provide protection against HPV52, the most common genotype found in this study. Nonetheless, Gardasil 9 would only cover $37 \%$ of the high-risk genotypes found. This has important implications for vaccine-strategic discussions. It should also send signals to vaccine manufacturers concerning future developments of new versions of the HPV vaccines. In the present study, we did not assess HPV vaccination status since no HPV vaccine had been introduced or administered within the National Health System.

This study has some limitations. Power calculation was applied for the HIV incidence in the parent study but not for the HPV ancillary study. We did not include an older cohort (aged $\geq 25$ years) for comparison of circulating HPV genotypes due to funding restrictions. This must be considered in future studies to better describe the epidemiology of HPV infections in Mozambique. In our study, the questionnaire assessing sexual behavioural captured information about the number of sexual partners categorised into two variables only, either having one or more than one partner. This did not enable us to further analyse the influence of the number of sexual partners on HPV status. The low-HPV prevalence in the male population may have been related to the site and technique of sampling and we therefore recommend that additional studies in male populations, in Mozambique, are considered.

\section{CONCLUSION}

This study confirmed the high burden of HPV infections in young women in the Maputo city, Mozambique. The HPV prevalence was demonstrated to be associated with high-risk sexual behaviour, thus confirming the need to intensify sex education and STI prevention interventions. Additional studies involving other anatomic collection sites in men to confirm the comparatively low prevalence of HPV are needed. For vaccine-strategic discussions, it is important to consider that the current HPV vaccines only cover a proportion of prevailing HPV types in this area.

\section{Author affiliations}

${ }^{1}$ Instituto Nacional de Saúde, Maputo, Mozambique

${ }^{2}$ Division of Clinical Microbiology, Department of Laboratory Medicine, Karolinska Institutet, Huddinge, Sweden

${ }^{3}$ Eduardo Mondlane University, Maputo, Mozambique

${ }^{4}$ Department of Laboratory Medicine, Faculty of Medicine and Health, Örebro University, Örebro, Sweden

${ }^{5}$ Department of Oncology-Pathology, Karolinska Institutet, Stockholm, Sweden ${ }^{6}$ Department of Microbiology, Public Health Agency of Sweden, Stockholm, Sweden ${ }^{7}$ Department of Microbiology, Tumor and Cell Biology, Karolinska Institutet, Stockholm, Sweden

${ }^{8} \mathrm{Hospital}$ Central de Maputo, Maputo, Mozambique

Acknowledgements We would like to express our gratitude to the women and men who participated in this study. We also acknowledge the outstanding dedication of the staff at the youth clinic in Maputo Central Hospital and the staff at the Instituto Nacional de Saúde and Örebro University Hospital laboratories. 
Contributors EV, CN, KF, NO, IJ, SA conceived and designed the study. EV, NI, MK, $\mathrm{GL}-\mathrm{I}, \mathrm{NO}$ performed the study and experiments. EV, OA analysed the data. $\mathrm{EV}, \mathrm{CN}$, $\mathrm{SA}, \mathrm{OA}$, IJ, GL-I, KF, TR, NO, NI, MK wrote the paper.

Funding This study was supported by funding from The Regional HIV/AIDS Team for Africa, Embassy of Sweden, Lusaka jointly funded by Sweden and Norway (Sida contribution number 2150012801).

Disclaimer Funders had no role in study design, data collection and analysis, decision to publish, or preparation of the manuscript.

Competing interests None declared.

Patient consent Obtained.

Ethics approval National Health Bioethics Committee of Mozambique and the Regional Ethical Review Board Uppsala.

Provenance and peer review Not commissioned; externally peer reviewed.

Data sharing statement Extra data are available by emailing EV (ednaviegas@ gmail.com).

Open Access This is an Open Access article distributed in accordance with the Creative Commons Attribution Non Commercial (CC BY-NC 4.0) license, which permits others to distribute, remix, adapt, build upon this work non-commercially, and license their derivative works on different terms, provided the original work is properly cited and the use is non-commercial. See: http://creativecommons.org/ licenses/by-nc/4.0/

(c) Article author(s) (or their employer(s) unless otherwise stated in the text of the article) 2017. All rights reserved. No commercial use is permitted unless otherwise expressly granted.

\section{REFERENCES}

1. Human papillomavirus and related diseases Report Africa. Barelona, Spain: Information Centre on HPV and Cervical Cancer, 2015.

2. Human papillomavirus and related diseases Report World. Barcelona, Spain: Information Centre on HPV and Cervical Cancer, 2015.

3. Internacional Human Papillomavirus Reference Center: Karolinska Institutet, 2015. Available from. http://www.hpvcenter.se/html/ refclones.html.

4. International Agency for Research on Cancer. IARC monographs on the evaluation of carcinogenic risks to humans. 90-100, 2012.

5. Muñoz N, Bosch FX, de Sanjosé S, et al. International Agency for Research on Cancer Multicenter Cervical Cancer Study Group. Epidemiologic classification of human papillomavirus types associated with cervical Cancer. N Engl J Med 2003;348:518-27.

6. Santos-López G, Márquez-Domínguez L, Reyes-Leyva J, et al. [General aspects of structure, classification and replication of human papillomavirus]. Rev Med Inst Mex Seguro Soc 2015;53 Suppl 2:S166-71.

7. Crow JM. HPV: The global burden. Nature 2012;488:S2-S3.

8. Guideline on when to start antiretroviral therapy and on pre-exposure prophylaxis for HIV. Geneva, Switzerland: World Health Organization 2015.

9. Asiaf A, Ahmad ST, Mohammad SO, et al. Review of the current knowledge on the epidemiology, pathogenesis, and prevention of human papillomavirus infection. Eur J Cancer Prev 2014;23:206-24.

10. Menéndez $\mathrm{C}$, Castellsagué $\mathrm{X}$, Renom $\mathrm{M}$, et al. Prevalence and risk factors of sexually transmitted infections and cervical neoplasia in women from a rural area of southern Mozambique. Infect Dis Obstet Gynecol 2010;2010:1-9.

11. Ginindza TG, Dlamini X, Almonte M, et al. Prevalence of and associated risk factors for high risk human papillomavirus among sexually active women, Swaziland. PLoS One 2017;12:e0170189.

12. Ebrahim S, Mndende XK, Kharsany AB, et al. High burden of human Papillomavirus (HPV) Infection among young women in KwaZuluNatal, South Africa. PLoS One 2016;11:e0146603.

13. Castellsagué $X$, Klaustermeier J, Carrilho C, et al. Vaccine-related HPV genotypes in women with and without cervical Cancer in Mozambique: burden and potential for prevention. Int $J$ Cancer 2008;122:1901-4.

14. Lorenzoni C, Vilajeliu A, Carrilho C, et al. Trends in Cancer incidence in Maputo, Mozambique, 1991-2008. PLoS One 2015;10:e0130469.
15. Carrilho C, Cirnes L, Alberto M, et al. Distribution of HPV infection and tumour markers in cervical intraepithelial neoplasia from cone biopsies of Mozambican women. J Clin Pathol 2005;58:61-8.

16. Carrilho $\mathrm{C}$, Gouveia $\mathrm{P}$, Cantel $\mathrm{M}$, et al. Characterization of human papillomavirus infection, P53 and Ki-67 expression in cervix Cancer of Mozambican women. Pathol Res Pract 2003;199:303-11.

17. Castellsagué X, Menéndez C, Loscertales MP, et al. Human papillomavirus genotypes in rural Mozambique. Lancet 2001;358:1429-30.

18. Naucler P, Mabota da Costa F, da Costa JL, et al. Human papillomavirus type-specific risk of cervical Cancer in a population with high human immunodeficiency virus prevalence: case-control study. J Gen Virol 2011;92:2784-91.

19. Pitisuttithum $P$, Velicer $C$, Luxembourg A. 9-Valent HPV vaccine for cancers, pre-cancers and genital warts related to HPV. Expert Rev Vaccines 2015;14:1405-19.

20. Viegas EO, Tembe N, Macovela E, et al. Incidence of HIV and the prevalence of HIV, hepatitis B and syphilis among youths in Maputo, Mozambique: a cohort study. PLoS One 2015;10:e0121452.

21. Pista A, Verdasca N, Oliveira A. Clinical performance of the CLART human papillomavirus 2 assay compared with the hybrid capture 2 test. J Med Virol 2011;83:272-6.

22. Rebolj M, Lynge E, Ejegod D, et al. Comparison of three human papillomavirus DNA assays and one mRNA assay in women with abnormal cytology. Gynecol Oncol 2014;135:474-80.

23. Watson-Jones D, Baisley K, Brown J, et al. High prevalence and incidence of human papillomavirus in a cohort of healthy young African female subjects. Sex Transm Infect 2013;89:358-65

24. Olesen TB, Munk C, Christensen J, et al. Human papillomavirus prevalence among men in sub-Saharan Africa: a systematic review and meta-analysis. Sex Transm Infect 2014;90:455-62.

25. Aguilar LV, Lazcano-Ponce E, Vaccarella S, et al. Human papillomavirus in men: comparison of different genital sites. Sex Transm Infect 2006;82:31-3.

26. Giuliano AR, Nielson CM, Flores R, et al. The optimal anatomic sites for sampling heterosexual men for human papillomavirus (HPV) detection: the HPV detection in men study. J Infect Dis 2007;196:1146-52

27. Smelov V, Eklund C, Bzhalava D, et al. Expressed prostate secretions in the study of human papillomavirus epidemiology in the male. PLoS One 2013;8:e66630.

28. Bruni L, Diaz M, Castellsagué X, et al. Cervical human papillomavirus prevalence in 5 continents: meta-analysis of 1 million women with normal cytological findings. J Infect Dis 2010;202:1789-99.

29. Ho GY, Bierman R, Beardsley L, et al. Natural history of cervicovaginal papillomavirus infection in young women. $N$ Engl J Med 1998;338:423-8.

30. Ramanakumar AV, Naud P, Roteli-Martins CM, et al. Incidence and duration of type-specific human papillomavirus infection in high-risk HPV-naïve women: results from the control arm of a phase II HPV16/18 vaccine trial. BMJ Open 2016;6:e011371.

31. Orlando G, Fasolo M, Mazza F, et al. Risk of cervical HPV infection and prevalence of vaccine-type and other high-risk HPV types among sexually active teens and young women (13-26 years) enrolled in the VALHIDATE study. Hum Vaccin Immunother 2014;10:986-94.

32. Ahdieh L, Klein RS, Burk R, et al. Prevalence, incidence, and type-specific persistence of human papillomavirus in human immunodeficiency virus (HIV)-positive and HIV-negative women. $J$ Infect Dis 2001;184:682-90.

33. Clifford GM, Gonçalves MA, Franceschi S. HPV and HIV Study Group. Human papillomavirus types among women infected with HIV: a meta-analysis. AIDS 2006;20:2337-44.

34. McDonald AC, Tergas Al, Kuhn L, et al. Distribution of human papillomavirus genotypes among HIV-Positive and HIV-Negative women in Cape Town, South Africa. Front Oncol 2014:4:48.

35. Williamson AL. The interaction between human immunodeficiency virus and human papillomaviruses in heterosexuals in Africa. J Clin Med 2015;4:579-92.

36. Levi JE, Kleter B, Quint WG, et al. High prevalence of human papillomavirus (HPV) infections and high frequency of multiple HPV genotypes in human immunodeficiency virus-infected women in Brazil. J Clin Microbiol 2002;40:3341-5.

37. Malagón T, Drolet M, Boily MC, et al. Cross-protective efficacy of two human papillomavirus vaccines: a systematic review and metaanalysis. Lancet Infect Dis 2012;12:781-9. 